\title{
La cultura organizacional y la efectividad de los programas de maestría
}

\author{
Eduardo Escalante Gómez ${ }^{1^{*}}$
}

1. Instituto de Investigaciones, Facultad de Psicología, Universidad del Aconcagua, Mendoza, Argentina.

\section{RESUMEN}

La finalidad de este estudio es investigar algunos factores de la organización que contribuyen al éxito de los alumnos de maestría. Se emplea una investigación basada en cuestionarios aplicados a alumnos en su etapa final de la tesis, en orden a estudiar la relación entre la orientación cultural de organización y la efectividad de los programas de maestría. El estudio reveló que la cultura de Clan se relaciona considerablemente con la efectividad de los programas de maestría. Se formulan algunas sugerencias a partir de los hallazgos para la organización y para futuras investigaciones.

Palabras claves: cultura organizacional, efectividad.

\section{INTRODUCCIÓN}

La investigación surge debido al alto número de alumnos de maestría que no logran finalizar sus tesis. Esta falta de efectividad organizacional se podría deber a falta de liderazgo académico, incumplimiento de las expectativas de los alumnos, falta de sistemas de apoyo investigativo, relaciones débiles entre directores de tesis y alumnos, entre otras. Esta temática toma especial relevancia dada la gran cantidad de programas de maestría. Medir este tipo de cuestiones, corresponde a realizar un estudio de la cultura organizacional en la que se realiza este tipo actividad académica.

La literatura permite indicar que se trata de un fenómeno que surge de la interacción con otras personas y se puede ver como un conjunto de estructuras, rutinas, reglas y normas que guían y restringen el comportamiento ${ }^{[1]}$. A través de la cultura, se pretende generar identidad organizacional, lograr el compromiso de sus miembros, generar estilos institucionales, entre otros factores.

Aunque la cultura se puede definir de distintas maneras, en el contexto académico, se refiere a ciertos valores y prácticas que se intenta que formen parte de la institucionalidad universitaria.

La literatura revela que la cultura, es uno de las pocas áreas donde se ha identificado un efecto de gran trascendencia en el desempeño de las organizaciones. El estudio de la cultura organizacional nos permite entender esa trascendencia. Sin embargo, existen algunas áreas no estudiadas como la efectividad de la formación de posgrado que se traduce en que los alumnos logren terminar exitosamente sus tesis .
Entender la cultura organizacional[ ${ }^{[2]}$ puede ser útil entre otras cosas, para que los administradores universitarios conciban la forma de mejorar la eficacia de su organización, para entender por qué existen dificultades en la coordinación e integración de procesos en las organizaciones ${ }^{[3]}$.

La cultura de la universidad se puede pensar como la personalidad de la organización, por lo cual poseen características distintivas que se correlacionan fuertemente con sus culturas ${ }^{[4]}$. En la actualidad, quienes dirigen las universidades están más concientes del concepto de cultura y su rol significativo en el desarrollo institucional.

A diferencia de otras organizaciones, las universidades tienen metas que a veces son difíciles de medir ${ }^{[5,6]}$. Constituyen una red compleja donde la tarea de sus administradores es relacionar sus distintos componentes, lo cual muchas veces resulta difícil y es por ello que se ha señalado que estaríamos en presencia de un proceso que ha sido definido como "flojedad estructural".

El entorno universitario ha cambiado considerablemente, con transformaciones significativas en la docencia, investigación, y administración en los últimos años ${ }^{[7]} \mathrm{con}$ un extraordinario predominio de modelos de gestión y acreditación que han derivados en serios escrutinios de su quehacer, generando grandes interrogantes sobre lo que ha ocurrido en ellas históricamente. Esto hace que puedan existir conflictos entre la cultura universitaria y las acciones de la jerarquía, lo cual puede resultar en alienaciones, desorganización, y confusión. 
El cambio organizacional debería incluir no solamente las estructuras y procesos sino también la cultura misma de la organización. Según Bennis, Spreitzer, y Cummings ${ }^{[8]}$, uno tiene que adherir simultáneamente a los símbolos de la tradición y la estabilidad y revisión del cambio para ser un agente efectivo de cambio.

Para detectar y explorar las características de cada cultura organizacional, se han desarrollado varios modelos. Cada uno ha incluido una gran variedad de dimensiones y atributos para medir la cultura organizacional. Algunos autores estiman que lo principal sería estudiar la congruencia y la solidez cultural; otros autores consideran que habría que centrarse en una dimensión fuerte-débil y en una dimensión interna-externa; y otros apuntan a entender la cultura organizacional a partir de la orientación a la gente (participativo versus no participativo) y respuesta al ambiente (reactivo versus proactivo).

El modelo de investigación empleado examina la relación entre la Cultura organizacional (Clan, Jerárquica, Adhocrática, y de Mercado) con la efectividad de los posgrados (Dictado de los Módulos, Expectativas Personales Cumplidas, Apoyo Académico Recibido, Orientación Investigativa, Logística Institucional, Revisiones Oportunas, Relación Director de Tesis - Alumno, Acceso a Documentación). Al análisis se incorporan algunas variables intermedias (sexo, edad, campo disciplinario).

En este estudio se adoptó el Competing Values Framework (CVF). Este referente teórico identifica 4 tipos de cultura organizacional, Clan, Jerárquica, Adhocrática, y de Mercado. Concretamente se usó el instrumento denominado OCAI (Organizational Culture Assessment Instrument, Instrumento de Caracterización de la Cultura Organizacional ${ }^{[9]}$.

La cultura organizacional tipo Clan se caracteriza por la interacción de la personas en un ambiente relativamente familiar. El tipo de liderazgo es "cercano" o "próximo" y se basa en la tradición y la lealtad, con un fuerte compromiso de sus miembros, lo que permite cohesión y alta moral. Además, se caracteriza por el consenso y la participación.

La cultura organizacional tipo Adhocracia se caracteriza por sus rasgos creativos, dinámicos en una ambiente de emprendimiento. El liderazgo es innovador y estimula la iniciativa individual y libertad intelectual.
La cultura organizacional tipo Jerarquizada se caracteriza por un grado considerable de estructuración y formalización basado en normas y procedimientos. Esto permite mantener a la organización cohesionada. De ello deriva que la preocupación fundamental del liderazgo sea la estabilidad y la eficacia.

La cultura organizacional tipo Mercado se caracteriza por su orientación a los resultados, y la calidad de lo que se realiza. Los niveles de competividad son altos dada la preocupación por el éxito y la reputación.

\section{DISEÑO METODOLÓGICO}

El diseño metodológico de esta investigación incluyó las siguientes variables: variable independiente: Tipo de cultura organizacional (Clan, Adhocracia, Jerárquica, de Mercado); variable dependiente: Efectividad de los programas de maestrías; y variables intermedias: sexo, edad, campo disciplinario.

La hipótesis investigativa es: Existe una relación significativa entre la cultura organizacional y la efectividad de los programas de maestría medida mediante ocho indicadores.

El universo total de alumnos de maestría de las universidades incluidas en el estudio es de 270. Un sesenta por ciento participan en programas de universidades públicas y un cuarenta por ciento en universidades privadas. La muestra estuvo conformada por 125 alumnos de maestría en la fase final de desarrollo de la tesis, 50 alumnas (40\%) y 75 alumnos (60\%); cuyas edades son: Menores de 30 años, 9 alumnos, entre 30 y 40 años, 100 alumnos, y mayores de 40 años, 16 alumnos. En términos de origen del campo disciplinario, 80 alumnos son de Ciencias Sociales y 45 alumnos de Ingeniería.

Para seleccionar el instrumento de medición de la cultura organizacional, se tomó en consideración la existencia de una metodología de análisis para diagnosticar la cultura institucional y es por ello que se utilizó el OCAI (Organizational Culture Assessment Instrument) que consta de 6 elementos: 1. Características dominantes, 2. Liderazgo Organizacional, 3. Administración del personal, 4. Unión de la organización, 5. Énfasis estratégico y 6 . Criterios de éxito. Cada uno de estos elementos presenta 4 alternativas: A, B, C y D. Para cada elemento se tiene 100 puntos, estos 100 puntos se deben distribuir entre las 4 alternativas. 
Para calcular la puntuación total alcanzada por cada una de las opciones $A, B, C$ y $D$, se deben sumar todos los puntos obtenidos en cada una de las opciones y dividir la suma entre 6 , es decir, calcular el promedio.

La efectividad de los programas de maestría se midió a través de un cuestionario con ocho indicadores: Dictado de los Módulos, Expectativas Personales Cumplidas, Apoyo Académico Recibido, Orientación Investigativa, Logística Institucional, Revisiones Oportunas, Relación Director de Tesis - Alumno, Acceso a Documentación. Para cada elemento se tiene 100 puntos, estos 100 puntos se deben distribuir entre las 4 alternativas. Para calcular la puntuación total alcanzada por cada una de las opciones A, B, C y D, se deben sumar todos los puntos obtenidos en cada una de las opciones y dividir la suma entre 8, es decir, calcular el promedio.

Para la creación de cada indicador de la efectividad de los programas de maestría, se elaboraron varias matrices. A modo de ejemplo, el modelamiento de las cuatro alternativas del indicador Relación Director de Tesis - Alumno tuvo como referencia la tabla 1 , de la cual se derivaron las 4 alternativas: A. Básico, B. Emergente, C. Intermedio, y D. Experto.

Se empleo el mismo criterio referido más arriba, si para este ítem, la persona piensa que la alternativa A es muy diferente a la situación actual, las alternativas B y $\mathrm{C}$ son relativamente similares, y la alternativa $D$ es difícilmente similar, se podría asignar 15 puntos a A, 40 puntos para $B$ y para $C$, y 5 puntos para $D$. Hay que asegurar que se totalizan 100 puntos para cada ítem.

Tabla 1 - Generación del indicador Los datos se analizaron en SPSS 15.0 y Amos 16.

\begin{tabular}{|c|c|c|c|}
\hline & Emergente & Intermedio & Experto \\
\hline \multirow{2}{*}{$\begin{array}{l}\text { Relación } \\
\text { Director } \\
\text { de Tesis } \\
\text {-Alumno }\end{array}$} & $\begin{array}{c}\text { Poca } \\
\text { participación } \\
\text { con el tesista }\end{array}$ & $\begin{array}{c}\text { Genera } \\
\text { oportunidades } \\
\text { para que el tesista } \\
\text { realice trabajo } \\
\text { autónomo }\end{array}$ & $\begin{array}{c}\text { Orienta al } \\
\text { tesista al } \\
\text { trabajo } \\
\text { auto-dirigido }\end{array}$ \\
\hline & Cómo & Qué-Cómo & $\begin{array}{l}\text { Qué-cómo-Por } \\
\text { qué }\end{array}$ \\
\hline
\end{tabular}

\section{RESULTADOS Y DISCUSIÓN}

Se construyeron los perfiles de la cultura organizacional, a partir de los análisis de los puntajes promedio de las cuatro alternativas ( $A=$ Clan, $B=$ Adhocracia, $C=$ Mercado, $D=$ Jerarquía).

En la Tabla 2, se puede apreciar que al diagnosticar la orientación cultural actual, la cultura más común es la orientación de mercado seguida por la jerárquica. Por otra parte, para la cultura preferida, la más elegida es la de clan, seguida por la jerárquica. Todos los puntajes promedios son estadísticamente significativo $(F: 15,7$, $P<0.0001$; F: 7,02, $P<0.001)$.

Al aplicar la prueba $t$ test se pudo observar que bajo la actual orientación cultural, la diferencia entre la orientación de mercado y la orientación jerárquica no es estadísticamente significativa. Al estudiar la cultura preferida, la diferencia entre las orientaciones de mercado y adhocracia, se detectó que no es estadísticamente significativa, no obstante, la diferencia entre la orientación cultural de clan y las otras orientaciones es significativa.

Tabla 2. Orientaciones culturales de los alumnos de posgrado - Puntajes promedios

\begin{tabular}{ccc}
\hline $\begin{array}{c}\text { Orientación } \\
\text { Cultural }\end{array}$ & Orientación Actual & Orientación Preferida \\
\hline Clan & 24,5 & 30,3 \\
Adhocracia & 15,4 & 21,3 \\
Mercado & 33,9 & 20,6 \\
Jerarquía & 27,2 & 25,4 \\
Cómo & Qué-Cómo & Qué-cómo-Por qué \\
\hline
\end{tabular}

Como se puede interpretar a partir del planteamiento de Cameron y Quinn ${ }^{[9]}$, la descripción de la cultura de Clan es indicativa de que las unidades académicas, en este caso, el programa de maestría, es un lugar amistoso, donde los académicos comparten con sus alumnos. Quienes están a cargo se perciben como autoridades acogedoras que contienen.

En la cultura de Clan, la institución se sostiene mediante la lealtad y la tradición, y el compromiso es alto. Lo cual demanda el apoyo a los logros de aprendizaje de los alumnos a través de procesos de orientación, tutoría y apoyo. Este es el tipo de cultura que los alumnos de posgrado prefieren para alcanzar el éxito en sus tesis y su desarrollo personal. Los alumnos esperan que exista un entorno colaborativo, centrado en el aprendizaje en el cual los académicos especializados orienten e integren docencia e investigación.

Respecto de la variable independiente (cultura organizacional) y las variables intermedias (sexo, edad, campo disciplinario). No se detectaron diferencias significativas respecto del sexo $(t=-0,316$; sig. 0,753$)$ y de la edad recodificada $(F=2,743$; sig. 0,192$)$, pero sí respecto del campo disciplinario $(t=-3,229$; sig. 0,002). 
La cultura organizacional dominante al considerar la situación actual, según los alumnos de maestrías en Ingeniería es de tipo Jerárquica y de Clan respecto de la situación preferida. Al considerar los alumnos de maestría en Ciencias Sociales, se puede apreciarse que el tipo de cultura dominante percibida en la situación actual, es la de Mercado, mientras que el tipo de cultura en la situación deseada es la Clan. En la tabla 3 se pueden observar los puntajes totales ponderados de las medidas de efectividad.

Tabla 3: Medidas de Efectividad - Puntajes totales Ponderados

\begin{tabular}{ccc}
\hline $\begin{array}{c}\text { Medidas de } \\
\text { Efectividad }\end{array}$ & $\begin{array}{c}\text { Puntajes totales } \\
\text { ponderados }\end{array}$ & Efectividad \\
\hline $\begin{array}{c}\text { Dictado de los } \\
\text { Módulos }\end{array}$ & 294.0 & Apropiada \\
\hline $\begin{array}{c}\text { Expectativas } \\
\text { Personales } \\
\text { Cumplidas }\end{array}$ & 281.0 & Apropiada \\
\hline $\begin{array}{c}\text { Apoyo Académico } \\
\text { Recibido }\end{array}$ & 268.0 & Intermedia \\
\hline $\begin{array}{c}\text { Orientación } \\
\text { Investigativa }\end{array}$ & 264.0 & Intermedia \\
\hline $\begin{array}{c}\text { Logística } \\
\text { Institucional }\end{array}$ & 261.0 & Baja \\
\hline $\begin{array}{c}\text { Revisiones } \\
\text { Oportunas }\end{array}$ & 253.0 & Baja \\
\hline $\begin{array}{c}\text { Relación Director } \\
\text { de Tesis - Alumno }\end{array}$ & 245.0 & Baja \\
\hline $\begin{array}{c}\text { Acceso a } \\
\text { Documentación }\end{array}$ & 243.0 & \\
\hline
\end{tabular}

Están ordenados según orden de importancia. Se detecta conformidad con el Dictado de los Módulos y Expectativas Personales Cumplidas, no obstante, los indicadores Revisiones Oportunas, Relación Director de Tesis - Alumno, Acceso a Documentación tienen puntuaciones que son bajas y revelan indicadores críticos o de relativamente baja calidad. Globalmente, se percibe una situación actual relativamente efectiva en sus aspectos organizativos, en especial, la organización de las materias, no obstante, se detectan brechas entre lo actual y lo preferido respecto de los indicadores mencionados.

Se calculó la confiabilidad del cuestionario de Caracterización de la Cultura Organizacional, para lo cual se usó el Alfa de Cronbach. La Tabla 4 muestra la confiabilidad de los constructos. Para explorar si existía un conjunto de supuestos causales entre variables, se empleó el análisis de senderos (path análisis). Aunque es preciso aclarar que este tipo de análisis no es un procedimiento para demostrar la existencia de causalidad, en forma definitiva[10].
Tabla 4. Confiabilidad de los constructos

\begin{tabular}{cc}
\hline Constructos & Alfa de Cronbach \\
\hline Clan & 0.793 \\
\hline Adhocracia & 0.683 \\
\hline Mercado & 0.712 \\
\hline Jerárquica & 0.830 \\
\hline $\begin{array}{c}\text { Efectividad del } \\
\text { posgrado }\end{array}$ & 0,687 \\
\hline
\end{tabular}

Este tipo de análisis se considera una extensión del modelo de regresión. A partir de la matriz de correlación se comparan dos o más modelos. El coeficiente obtenido es un coeficiente estandarizado de regresión (beta), que muestra el efecto directo de una variable independiente en una variable dependiente en el modelo de sendero (path model).

La tabla 5 muestra los coeficientes de sendero (path coefficients) y los valores $t$ para relaciones significativas. Respecto del resto de relaciones de la Efectividad del posgrado y el resto de las orientaciones, los valores no fueron significativos: Adhocracia (coeficiente de sendero $=0,17$ y 0,12; valor $T=0,094$ y 0,113); Jerárquica (coeficiente de sendero $=0,15$ y 0,11 ; valor $T=1,784$ y 0,237); y Mercado (coeficiente de sendero=0,13 y 0,10; valor $T=1,089$ y 0,096).

Tabla 5. Confiabilidad de los constructos

\begin{tabular}{ccc}
\hline Hipótesis & $\begin{array}{c}\text { Coeficientes de } \\
\text { Sendero (path } \\
\text { coeficientes) }\end{array}$ & Valor T \\
\hline $\begin{array}{c}\text { Efectividad } \\
\text { del posgrado- } \\
\text { Orientación actual: } \\
\text { Clan }\end{array}$ & 0.58 & $4.15^{\star * *}$ \\
\hline $\begin{array}{c}\text { Efectividad } \\
\text { del posgrado } \\
\text {-Orientación } \\
\text { preferida: Clan }\end{array}$ & 0.49 & $3.35^{* *}$ \\
\hline
\end{tabular}

${ }^{* *} p<0.05,{ }^{* * *} p<0.01$

Basados en la Tabla 5, la hipótesis investigativa que establece que existe una relación significativa entre la cultura organizacional y la efectividad de los programas de maestría medida por ocho indicadores, se acepta para la orientación cultural de Clan.

La cultura de Clan enfatiza la importancia de la participación, cohesión, valores compartidos, compromiso y alta moral. La orientación de cultura de Clan se asocia a la efectividad del programa de posgrado, esto es, finalización y aprobación de la tesis, cumplimientos de expectativas, adecuadas relaciones director de tesis-alumno, etc. 


\section{CONCLUSIONES}

Se empleo un modelo de investigación basado en cuestionarios para estudiar las relaciones entre las orientaciones de la cultura organizacional y la efectividad de los programas de maestría. Se halló que la cultura de Clan está asociada significativamente a la efectividad de los programas de maestría.

Este estudio contribuye a revelar el papel de la cultura organizacional y el éxito de los programas de maestría. La cultura de Mercado basada en resultados, la cultura Jerárquica caracterizada por las reglas y procedimientos, no parece contribuir a la efectividad de los programas de posgrado.

Es interesante detectar que la cultura Adhocrática que asume la innovación, creatividad y comportamiento visionario no juega un papel en la efectividad de los programas de maestría estudiados.

Estos resultados indicarían que quienes dirigen los programas de posgrado, deberían facilitar la cohesión efectiva, facilitar las relaciones interpersonales efectivas entre directores de tesis y alumnos de maestría, y comprometerse con los alumnos para la concreción de sus proyectos de tesis y su defensa final exitosa. Además, si se quiere lograr un posicionamiento (interno y externo) efectivo, se requiere mayores niveles de innovación en el proceso docente y en las temáticas y desarrollo de las tesis.

\section{REFERENCIAS BIBLIOGRÁFICAS}

1. Schein, E. H. (2004), "Organizational Culture and Leadership", Jossey-Bass, San Francisco, CA.

2. Hofstede, G. (2001), Culture's Consequences: Comparing Values, Behaviors, Institutions and Organizations across Nations, Sage, Thousand Oaks, CA.

3. Kotter J. P., and Heskett, J. L. (1992), Corporate Culture and Performance, New York: Free Press.

4. Bartell, M. (2003). Internationalization of universities: a university culture-based framework. Higher Education, 45, 43-70

5. Baldridge, J.V., Curtis, D.V., Ecker, G., \& Riley, G.L. (1978). Policy Making and Effective Leadership.San Francisco: Jossey - Bass.
6. Birnbaum, R. (1988). How Colleges Work: The Cybernetics of Academic Organization and Leadership.San Francisco: Jossey-Bass.

7. Cohen, D.W. (1997). Understanding the globalization of scholarship, in Peterson, M.W., Dill, D.D., Mets, L. A. and Associates (eds.), Planning and Management for a Changing Environment: A Handbook onRedesigning Postsecondary Institutions. San Francisco: Jossey-Bass, 548-562.

8. Bennis, W.G., Spreitzer, G.M. and Cummings, T. (2001). The Future of Leadership. John Wiley \& Sons, Inc.

9. Cameron, K.S. \& Quinn, R.E. (1999). Diagnosing and Changing Organizational Culture Based on the Competing Values Framework. Addison-Wesley Publishing Company, Inc.

10. Briones, Guillermo (1996), Investigación Cuantitativa en las Ciencias Sociales. Arfo Editores e Impresores Ltda, Bogotá. 